\title{
A Thrombectomy Model Based on Ex Vivo Whole Human Brains
}

(D) Y. Liu, (D).L.A. Larco, (D) S.I. Madhani, (D)A.H. Shahid, (D).A. Quinton, (DR. Kadirvel, (DD.F. Kallmes, (D)W. Brinjikji, and

(iD) L.E. Savastano

it

\section{ABSTRACT}

BACKGROUND AND PURPOSE: The persistent challenges in thrombectomy for large-vessel occlusion, such as suboptimal complete recanalization and first-pass effect imply an insufficient understanding of the artery-clot-device interaction. In this study, we present a thrombectomy model using fresh human brains, which can capture the artery-clot-device interaction through concurrent transmural and angiographic visualizations.

MATERIALS AND METHODS: Fresh nonfrozen whole adult human brains were collected and connected to a customized pump system tuned to deliver saline flow at a physiologic flow rate and pressure. Angiography was performed to verify the flow in the anterior-posterior and vertebrobasilar circulations and collaterals. Large-vessel occlusion was simulated by embolizing a radiopaque clot analog. Thrombectomy was tested, and the artery-clot-device interactions were recorded by transmural and angiographic videos.

RESULTS: Baseline cerebral angiography revealed excellent penetration of contrast in the anterior-posterior and vertebrobasilar circulations without notable arterial cutoffs and with robust collaterals. Small branches $(<0.5 \mathrm{~mm})$ and perforating arteries were consistently opacified with good patency. Three device passes were performed to achieve recanalization, with failure modes including elongation, fragmentation, and distal embolization.

CONCLUSIONS: This model enables concurrent transmural and angiographic analysis of artery-clot-device interaction in a human brain and provides critical insights into the action mechanism and failure modes of current and upcoming thrombectomy devices.

ABBREVIATIONS: LVO = large-vessel occlusion; VA = vertebral artery

$\mathrm{T}$

hrombectomy has been the standard of care for stroke with large-vessel occlusion (LVO), but clinical challenges such as suboptimal rates of first-pass effect and overall complete recanalization persist even after years of experience and development of new thrombectomy devices. ${ }^{1,2}$ Such challenges imply the need for improved next-generation devices optimized in more realistic thrombectomy models. Historically, preclinical testing of thrombectomy devices has relied heavily on benchtop models. Although phantoms reproduce the gross arterial geometry and enable easy

Received April 30, 2021; accepted after revision July 8.

From the Departments of Radiology (Y.L., R.K., D.F.K., W.B.) and Neurosurgery (J.L.A.L., S.I.M., A.H.S., L.E.S.) and Division of Anatomic Pathology (R.A.Q.), Mayo Clinic, Rochester, Minnesota.

This work was supported by the National Institutes of Health National Institute of Neurological Disorders and Stroke (NS105853).

Please address correspondence to Luis E. Savastano, MD, PhD, Department of Neurosurgery, Mayo Clinic, 200 First St SW, Rochester, MN 55905; e-mail:

Savastano.Luis@mayo.edu; @SavastanoLuisMD

- Indicates open access to non-subscribers at www.ajnr.org

D. Indicates article with supplemental online videos.

http://dx.doi.org/10.3174/ajnr.A7291 and reproducible testing of devices, they have oversimplified vascular anatomy and have much stiffer vessel walls compared with the human cerebral vasculature. ${ }^{3,4}$ Animal models have also been used to evaluate device performance; however, the visualization of device-clot-artery interaction is limited by fluoroscopy, and the arteries do not accurately represent the complex anatomy and hemodynamic conditions of human brains. ${ }^{5}$ A thrombectomy model that can realistically mimic the mechanical properties, anatomy, and blood flow of human cerebral vasculature is an unmet need.

In this study, we present a thrombectomy model using ex vivo whole human brains with unmodified cerebral vasculature and realistic flow conditions. This model allows concurrent direct optical and fluoroscopic examinations of the device-clot-artery interactions. The preparation of the brains and recreation of LVO will be described, followed by thrombectomy testing for model validation.

\section{MATERIALS AND METHODS Brain Preparation}

The reparation method of the brains has been previously disclosed. ${ }^{6}$ Briefly, after local institutional review board approval 


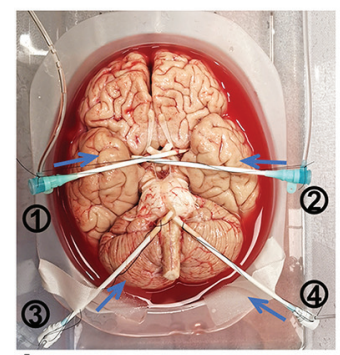

A

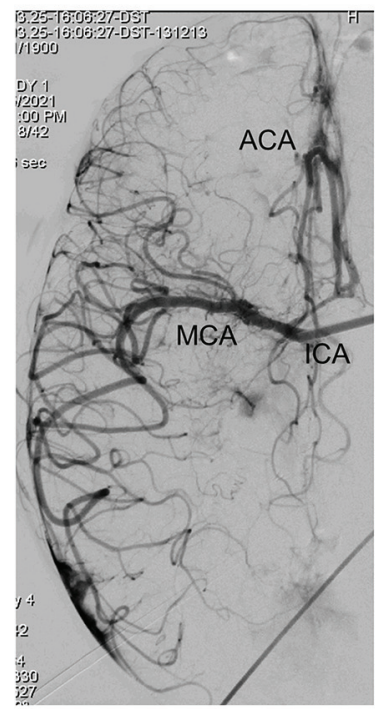

C

FIG 1. Ex vivo brain model flow setup and cerebral angiography. The bilateral ICAs and VAs of a fresh human cadaver brain were cannulated with sheaths to deliver saline solution and contrast (blue arrows) and catheters ( $A$ ). The sheaths were connected to a pump system and connected in parallel to an escape system, including a reservoir of saline hung at a height that mimics the back pressure, equivalent to the peripheral arterial resistance, that releases the cerebral pressure when an occlusion is created $(B)$. DSA of the right ICA in the late arterial phase in a pure ventrocaudal projection shows good opacification of the right MCA candelabra, right anterior cerebral artery (ACA), with cross-filling into the left ACA through a patent anterior communicating artery $(C)$. Occlusion of the proximal right MCA (asterisk) enhances the visualization of robust leptomeningeal collaterals (arrows) from the ACA into cortical MCA branches $(D)$. MCA indicates middle cerebral artery.

(20-002582), fresh nonfrozen whole adult human brains were collected during research-consented hospital postmortem examinations within 24 hours of the patient's death, and craniotomy was carefully performed to minimize damage to the brain and cerebral vessels. Brains with a history of head trauma, neurosurgical procedures, and stroke were excluded from this study. The arachnoid was then removed to expose the circle of Willis, including the anterior and posterior circulations. Postmortem clots were then removed by manual aspiration. The ICAs and vertebral arteries (VAs) were then cannulated with $8 \mathrm{~F}$ or $9 \mathrm{~F}$ femoral sheaths and tied with sutures (Fig 1A). The sheaths were then connected to a customized pump system to deliver $0.9 \%$ saline into the brain (Fig $1 B)$. The saline allows direct observation of the interactions between the clot, artery, and thrombectomy devices. The pump system was calibrated to deliver physiologically representative flow rates to the ICAs and VAs, and the pressure was tuned by adjusting the downstream flow resistance to recreate physiologic pressure. ${ }^{6}$ The flow rates were calibrated to infuse about $300 \mathrm{~mL} / \mathrm{min}$ for each ICA and $160 \mathrm{~mL} / \mathrm{min}$ for each vertebral artery. Then, using an 014 pressure-sensing guidewire (ComboWire XT; Philips Healthcare), we obtained intra-arterial pressures with a diastolic pressure around $80 \mathrm{~mm} \mathrm{Hg}$ and a systolic pressure of about $100 \mathrm{~mm} \mathrm{Hg}$. Outward pressure can be replicated by submerging the brain in a saline tank, and the saline height above the brain can be adjusted to match the intracranial pressure (5 to $20 \mathrm{~cm}$ of water). Baseline cerebral angiographies of the ICA and vertebrobasilar circulations were performed in anteroposterior and lateral projections by injecting $10 \mathrm{~mL}$ of contrast (iohexol, Omnipaque 300; GE Healthcare) in the sheath.

\section{Recreation of Large-Vessel Occlusion}

A fibrin-rich clot analog was made by mixing human plasma and red blood cells at a volume ratio of 19:2. To increase radiopacity, $0.5 \mathrm{~g}$ of barium sulfate powder was added per $10 \mathrm{~mL}$ of blood mixture. Higher amounts of barium sulfate can further increase the radiopacity but will make the clot analog more fragmentprone. ${ }^{4}$ Coagulation was induced by adding $1 \mathrm{~mL}$ of $5 \%$ calcium chloride and $10 \mu \mathrm{L}$ of $10 \mathrm{U} / \mathrm{mL}$ thrombin per $10 \mathrm{~mL}$ of blood mixture. ${ }^{7}$ To simulate LVO, we loaded clot analogs into a syringe and embolized them to the brain via a 0.088 -inch guide catheter (Neuron MAX; Penumbra) under physiologic pressure. This clot analog was selected to challenge current thrombectomy devices because fibrin-rich clots have been reported to be associated with more device passes, longer procedure times, and less favorable patient outcomes. ${ }^{8}$ Clot analogs with different histology and tensile properties can also be made by mixing human plasma and red blood cells with different volume ratios. ${ }^{7,9}$

\section{Thrombectomy Testing}

Thrombectomy was performed with a $6 \mathrm{~F}$ catheter (Sofia Plus; MicroVention) using the direct-aspiration technique or in combination with a stent retriever (Solitaire; Medtronic) using the Solumbra technique. These 2 techniques were selected because they are most often used for thrombectomy on the basis of a survey of neurointerventionalists. ${ }^{10}$ The aspiration catheter was navigated to the clot face over a 0.014 -inch guidewire (Synchro; Stryker) and a 0.027 -inch microcatheter (Phenom; Medtronic) under roadmap guidance. Aspiration was provided by a vacuum pump (Gomco 405; Allied Healthcare).

\section{RESULTS}

\section{Brain Model}

After a learning curve of about 8 brains (if the operator has extensive experience in cerebrovascular anatomic dissections), it is possible to consistently have an operational testing platform that replicates physiologic hemodynamic conditions and allows realtime visualization of the device-clot interaction in about $75 \%$ of all harvested brains. About one-fourth of the brains could not be used due to a variety of reasons including the following: 1) major leaks due to perforating vessel avulsion during the extraction process from the skull base or cortical damage with M4 branch transection with the bone saw (easy to see after connecting the arteries 

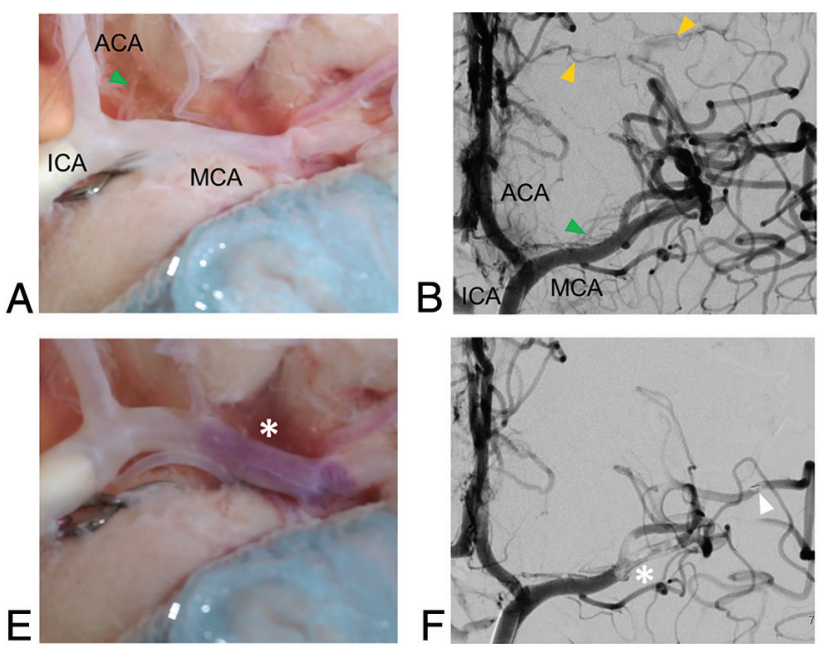
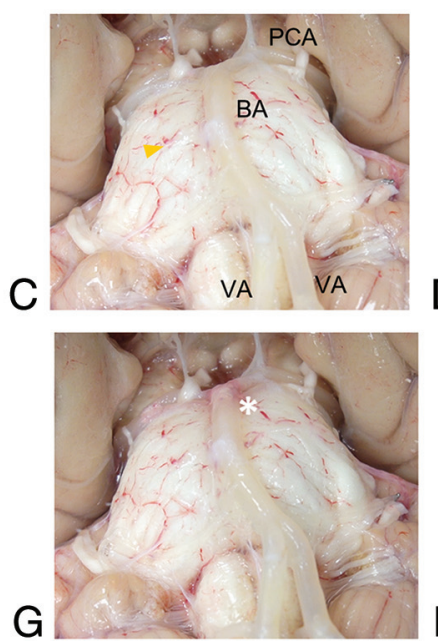
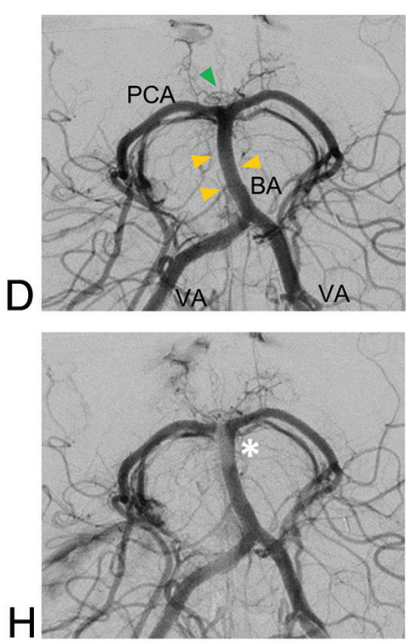

FIG 2. Concurrent direct transmural observation and DSA of the brain model pre- and post-LVO. Baseline transmural and angiographic visualization of the anterior circulation model $(A$ and $B$ ) includes ICA, MCA, anterior cerebral artery (ACA), lenticulostriate arteries (green triangle), and leptomeningeal cortical collaterals (yellow triangles). The posterior circulation model $(C$ and $D$ ) includes the vertebral arteries, the basilar artery with bifurcation into the posterior cerebral arteries, the thalamoperforating arteries (green triangle), and the pontine arteries (yellow triangles). Embolization of a radiopaque clot analog (asterisks, $E-H$ ) generally results in MCA bifurcation occlusion involving the lenticulostriate vessels $(E$ and $F$ ) and occasionally results in concurrent distal MCA branch occlusion (triangle in $F$ ) and occlusion of the basilar artery apex ( $G$ and $H$ ).

to the flow system); 2) thick circumferential atherosclerotic plaques that prevent transmural visualization; 3) the length of the paraclinoid ICA and V4 segment of the VA being too short to allow cannulization by the sheaths. About 10-15 thrombectomy passes can be tested for each brain (about 5 in each anterior circulation and 5 in the basilar artery), but that changes depending on the type of clot and device used. After the brain is harvested, preparation and testing need to be done in about 6 hours before the brain decays.

\section{Baseline Cerebral Angiography}

Cerebral angiography revealed excellent penetration of contrast in the ICA and the vertebrobasilar circulation both in the arterial and capillary phases without notable arterial cutoffs and occasional minimal extravasation. Small branches $(<0.5 \mathrm{~mm})$ and perforating arteries were consistently opacified by radiopaque contrast with good patency (Fig 1C). Interhemispheric collateral flow through the circle of Willis was identified. Robust leptomeningeal collaterals were also identified by creating a proximal MCA occlusion and following contrast flow from the anterior cerebral artery to the cortical MCA branches in the late arterial phase (Fig 1D).

\section{Concurrent Fluoroscopic and Transmural Visualization of Embolic Large-Vessel Occlusion}

Direct transmural visualization concurrent with angiography was feasible for both the ICA and the vertebrobasilar circulation (Figs $2 A-D$ ). The anterior circulation model provided visualization of the intracranial ICA system, including the lenticulostriate vessels and cortical leptomeningeal collaterals (Figs $2 A,-B$ ). The posterior circulation model revealed the vertebrobasilar system including the thalamoperforating and pontine arteries (Figs 2C, -D). Embolization of the radiopaque clot analog could be visualized in real-time by fluoroscopy (Online Video 1). Postembolization cerebral transluminal observation and angiography demonstrated LVO at the MCA bifurcation (Figs $1 E,-F$ ) and the basilar artery apex (Figs 1G, $-H$ ), which remained stable under physiologic flow and pressure. In the anterior circulation, postembolization contrast filling defects included the lenticulostriate arteries and distal cortical MCA branches, likely due to partial clot fragmentation and downstream migration (Fig $1 F$ ). In the posterior circulation, postembolization contrast filling defects included the pontine arteries and the thalamoperforating arteries (Fig 1H).

\section{Concurrent Fluoroscopic and Optical Visualization of the Recanalization Procedure}

The model allowed concurrent fluoroscopic and optical visualization through the arterial wall of the thrombectomy, greatly enhancing the analysis of device-clot interaction. For example, for an occlusion at the basilar apex, 3 device passes were performed to recanalize the parent artery. In the first pass (Online Video 2), the aspiration catheter was navigated to the clot face, and vacuum was then activated. Vacuum and device withdrawal are needed to overcome the resistance forces including the vessel wall friction and hydrodynamic forces in the antegrade direction. The clot corked the catheter tip and started to be pulled back with an initial length of $\mathrm{L}_{0}$ (Fig $3 A$ ). During pulling, the tensional load stretched the clot to a length of $\mathrm{L}_{1}$ (Fig $3 B$ ). As the clot was further pulled to enter the smaller VA, the increased resistance forces surpassed the device-clot integration force resulting in clot disengagement from the catheter (Fig 3C). The clot was then pushed downstream by the flow, resulting in a recurrent occlusion at the basilar artery apex. In the second pass (Online Video 3 ), as the clot was pulled into the VA, the tensional load elongated and weakened the clot and eventually caused clot fragmentation with distal embolization to the basilar artery apex. In the third pass (Online Video 4), the remaining clot fragment remained corked at the catheter tip and was pulled out without disengagement or fragmentation. Compared with the previous 2 passes, the clot fragment in the third pass had a smaller surface area and, 

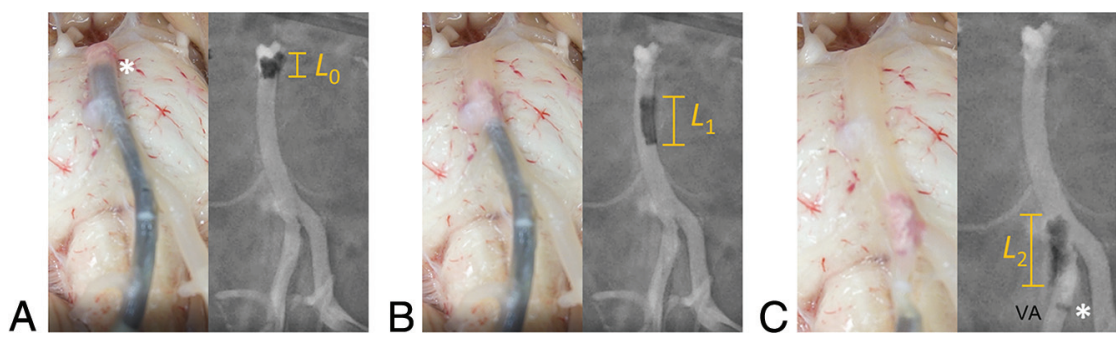

FIG 3. Concurrent transmural and angiographic visualization of a direct-aspiration thrombectomy for an embolic occlusion at the basilar apex. The aspiration catheter was navigated to the clot face, and the clot with an initial length of $L_{0}$ was corked by vacuum at the catheter tip (asterisk) (A). Device pullout elongated the clot to a length of $L_{1}$ and disengaged the clot from the basilar apex $(B)$. As the clot was pulled from the basilar artery into the right $V A$, it further elongated $\left(L_{2}\right)$ and was stripped away from the catheter tip (asterisk in C).

therefore, smaller frictional and hydrodynamic shear forces fighting against the catheter pull, leading to a smaller tensional load within the clot.

\section{DISCUSSION}

In this article, we introduced a human cadaveric model for cerebrovascular research with concurrent transmural and angiographic visualization and demonstrated its value for testing in LVO. Compared with our previous work in which thrombectomy testing in ex vivo brains was transmurally observed, ${ }^{6}$ the angiographic study presented here included baseline cerebrovascular anatomy in cadavers and the changes during and after thrombectomy, revealing important features such as the presence of leptomeningeal collaterals and the capacity to assess the patency of small branches and perforating arteries. In addition, the previously published model was able to only assess recanalization in the arterial segments exposed at the base of the brain (mostly the distal VA, basilar artery, and P1 and the ICA terminus with the proximal M1 and A1). The present model here introduced provides a much broader area of analysis, practically including the whole brain and the cerebral vasculature down to the cortical branches. In addition to a more clinically relevant and accurate scoring of the recanalization procedure, it may enable the testing of miniaturized thrombectomy devices in the removal of thrombi from distal branches to eloquent brain areas.

In addition to the human brain model herein described, another 3 main categories of thrombectomy models have been reported and reviewed in the literature: benchtop phantoms, ${ }^{11}$ in vivo animal models, ${ }^{12}$ and emerging computational models. ${ }^{13}$ Benchtop phantoms leverage recent advances in high-resolution medical imaging and 3D printing techniques to replicate patientspecific anatomy in a consistent fashion. This feature is advantageous in early device testing and benchmarking of different devices. Hemodynamics in the benchtop phantoms can also be customized to replicate physiologic conditions. Transparent phantoms also allow direct visualization of the device-clot interaction, though the "vascular" walls are much stiffer than human arteries (or totally rigid), lack submillimeter arteries, and do not adhere well to the clots. ${ }^{11}$ Animal models have been extensively used and are critical to test device safety. These models provide a more realistic device-artery-clot interaction, but it can only be visualized using fluoroscopy, with a limited resolution and frame rate. In addition, the blood flow in animal models is usually slower than that of the human cerebrum, resulting in an underestimation of hemodynamic force and events of distal embolization, and the 3D anatomic configuration is much simpler than the human cerebral vasculature. Finally, computational models may be promising to generate a realistic "virtual patient" model with characteristics otherwise difficult to be simultaneously mimicked in phantoms and animals such as anatomic tortuosity, hemodynamics, and vessel wall properties. However, the development of a realistic and validated biomechanical model continues to be a persisting challenge.

First, this model has the unmodified geometric complexity of the human cerebral vasculature, including a patent circle of Willis, small branches and perforating arteries, and functional leptomeningeal collaterals. These anatomic features are extremely important because the models used to date are mostly artificial benchtop phantoms that do not have submillimeter arteries. ${ }^{11}$ Animal models (eg, swine) do not fully emulate the human cerebral vasculature distribution or wall characteristics. ${ }^{3}$ Residual occlusion in small branching and perforating arteries is believed to be one of the reasons for poor neurologic outcome despite apparently "complete" recanalization, and this model may be of benefit to better characterize this problem and develop solutions. ${ }^{14}$

Second, the human brain model provides a realistic mechanical response of the arteries to the mechanical load of devices. This is critical to accurately test arterial deformation, stretching, collapse under vacuum, and even arterial injury such as perforation. Recently, mechanical thrombectomy devices were recalled from the market after multiple events of fatal catastrophic arterial ruptures. ${ }^{15}$ By comparison, benchtop phantoms have much stiffer and stronger walls, preventing arterial deformation, traction, and even injury, and the friction encountered is not realistic. ${ }^{3,11}$ Animal models do provide a more realistic environment and enable gross analysis of arterial injury, but recent publications have shown that human arteries differ both in architecture and mechanical strength compared with the extracranial arteries of animals, ${ }^{5}$ and the analysis of the response continues to be limited by the resolution and frame rate of fluoroscopy.

Third, this model allows concurrent fluoroscopy and direct transluminal observation of artery-clot-device interaction to understand the action mechanism and failure modes of thrombectomy devices. Neurointerventionalists are blind to the arterial wall response under vacuum and/or device pull; therefore, a model that "opens a window" for direct visualization has a large potential in research and improvement of technology. In this study, we have demonstrated that pulling back of thrombectomy devices can cause elongation, thinning, weakening, and possible fracturing of the clot, leading to distal embolization and residual occlusion. Although such a phenomenon has been reported in 
benchtop models, ${ }^{16}$ fidelity of this brain model is higher, and analysis is more comprehensive due to the more realistic vessel wall properties, including friction and strength. In addition, the realistic mechanical properties of the vessel wall make this model unique to reveal failure modes of device-artery interaction such as vessel traction and vessel collapse as shown previously. ${ }^{14}$ Pulling back the catheter with weak clot-catheter integration results in residual occlusions or iatrogenic embolization and is likely the main cause of low first-pass recanalization. We think that future aspiration technologies should aim to ingest clots in situ, proximal-to-distal, and at low vacuum power to prevent vessel collapse.

Although only embolic LVOs were presented in this study, we believe that this model can be adapted to recreate other neurovascular conditions, including medium and distal vessel occlusions (and test the performance of novel miniaturized thrombectomy devices), brain aneurysms, and intracranial atherosclerotic diseases.

We do acknowledge some limitations with this model that must be considered to extrapolate results to our patients. First, saline was used to enable direct transmural observation of the thrombectomy procedure, but the viscosity is lower than that of blood. Blood can be used to replicate the hydrodynamics, though this will limit direct transmural observation. Ideally, other colorless and transparent fluids that can simultaneously mimic the viscosity and friction of blood can be used, though such fluid has not been reported in the literature. Second, the brain is ex vivo, and biologic activities such as vasospasm and vascular tone cannot be captured. Third, the model as presented in this article does not include the tortuous path of the ICA through the skull base, though this feature could be implemented if needed by using appropriately shaped sheaths. Fourth, the degree of arterial deformation in this model could be potentially more pronounced given the absence of enclosing calvaria and skull base; therefore, extrapolation to patients needs to be considered with care. Last, variance among different brains is inevitable (for example in the size and shape of the circle of Willis), somehow limiting the reproducibility of results, and necessitates testing multiple brains to minimize bias. Overall, this is a proof-of-concept study, and further validation against clinical experience and other thrombectomy models are warranted.

\section{CONCLUSIONS}

The whole-human brain model with concurrent transmural and angiographic visualization of recanalization provides unsurpassed fidelity of the human cerebrovascular system and enables accurate analysis of artery-clot-device interaction in thrombectomy.

Disclosures: Yang Liu—UNRELATED: Stock/Stock Options: Endovascular Engineering. Ram Kadirvel—RELATED: Grant: National Institutes of Health, Comments: National Institutes of Health NS076491.* David F. Kallmes_UNRELATED: Board Membership: Triticum,* Comments: Advisory Board; Consultancy: NoNO, Vesalio, Minnetronix, ${ }^{*}$ Comments: Data and Safety Monitoring Board; Grants/Grants Pending: Balt, MicroVention, Medtronic, Neurogami, Monarch Medical, Insera Therapeutics, MIVI Neurovascular,* Comments: research support; Patents (Planned, Pending, or Issued): Balloon-Guide technology, Spine Augmentation technology*; Royalties: Balloon Guide Technology; Stock/Stock Options: Marblehead Medical, Conway Medical
Center, Superior Medical Experts. Luis E. Savastano-UNRELATED: Board Membership: President of Endovascular Engineering; Employment. Chief Medical Officer of VerAvanti; Stock/Stock Options: VerAvanti and Endovascular Engineering. *Money paid to the institution.

\section{REFERENCES}

1. Kleine JF, Wunderlich S, Zimmer C, et al. Time to redefine success? TICI 3 versus TICI 2 b recanalization in middle cerebral artery occlusion treated with thrombectomy. J NeuroIntervent Surg 2017;9:117-21 CrossRef

2. Abbasi M, Liu Y, Fitzgerald S, et al. Systematic review and metaanalysis of current rates of first pass effect by thrombectomy technique and associations with clinical outcomes. J Neurointerv Surg 2021;13:212-16 CrossRef Medline

3. Liu Y, Abbasi M, Arturo Larco JL, et al. Preclinical testing platforms for mechanical thrombectomy in stroke: a review on phantoms, invivo animal, and cadaveric models. J Neurointerv Surg 2021;13:81622 CrossRef Medline

4. Reddy A, Liu Y, Cockrum J, et al. Construction of a comprehensive endovascular test bed for research and device development in mechanical thrombectomy in stroke. J Neurosurg 2020;134:1190-97 CrossRef Medline

5. Ding Y, Fitzgerald S, Liu Y, et al. A novel rabbit thromboembolic occlusion model. J Neurointerv Surg 2021 Feb 11. [Epub ahead of print] CrossRef Medline

6. Gebrezgiabhier D, Liu Y, Reddy AS, et al. A human brain test bed for research in large vessel occlusion stroke. J Neurosurg 2021 Jan 22. [Epub ahead of print] CrossRef Medline

7. Fitzgerald S, Liu Y, Dai D, et al. Novel human acute ischemic stroke blood clot analogues for in-vitro thrombectomy testing. AJNR Am J Neuroradiol 2021;42:1250-57 CrossRef Medline

8. Jolugbo P, Ariëns RA. Thrombus composition and efficacy of thrombolysis and thrombectomy in acute ischemic stroke. Stroke 2021;52:1131-42 CrossRef Medline

9. Liu Y, Reddy AS, Cockrum J, et al. Standardized fabrication method of human-derived emboli with histologic and mechanical quantification for stroke research. J Stroke Cerebrovasc Dis 2020;29:105205 CrossRef Medline

10. Fargen KM, Arthur AS, Spiotta AM, et al. A survey of neurointerventionalists on thrombectomy practices for emergent large vessel occlusions. J Neurointerv Surg 2017;9:142-46 CrossRef Medline

11. Waqas M, Mokin M, Lim J, et al. Design and physical properties of 3-dimensional printed models used for neurointervention: a systematic review of the literature. Neurosurgery 2020;87:E445-53 CrossRef Medline

12. Herrmann AM, Meckel S, Gounis MJ, et al. Large animals in neurointerventional research: a systematic review on models, techniques and their application in endovascular procedures for stroke, aneurysms and vascular malformations. J Cereb Blood Flow Metab 2019;39:375-94 CrossRef Medline

13. Konduri PR, Marquering HA, van Bavel EE, et al; INSIST Investigators. In-silico trials for treatment of acute ischemic stroke. Front Neurol 2020;11:558125 CrossRef Medline

14. Liu Y, Gebrezgiabhier D, Reddy AS, et al. Failure modes and effects analysis of mechanical thrombectomy for stroke discovered in human brains. J Neurosurg 2021 June 4. [Epub ahead of print] CrossRef Medline

15. Majidi S, Bageac DV, Fayed I, et al. JET 7 XTRA Flex reperfusion catheter-related complications during endovascular thrombectomy. J Neurointerv Surg 2021;13:352-56 CrossRef Medline

16. Liu Y, Zheng Y, Reddy AS, et al. Analysis of human emboli and thrombectomy forces in large vessel occlusion stroke. J Neurosurg 2020;134:893-901 CrossRef Medline 